\title{
IMPACT OF THE DAMPER CHARACTERISTICS ON THE BEHAVIOR OF SUSPENSION SYSTEM AND THE WHOLE VEHICLE
}

\author{
Miloš Maljković*, Ivan Blagojević, Vladimir Popović, Dragan Stamenković \\ University of Belgrade, Faculty of Mechanical Engineering, Belgrade, Serbia
}

Damper asymmetry is a phenomenon for which there is no confirmed explanation in the literature, although it is present in almost all variants of telescopic dampers. This paper analyzes the influence of various characteristics of dampers, both symmetrical and asymmetrical, on the behavior of the suspension system and the whole vehicle. Different tests have been carried out, and they simulate the different types of vehicle movement that are present in exploitation. The simulation was performed in CarSim 8 software package for the vehicle with chosen characteristics. Appropriate conclusions were drawn on the basis of the conducted research, i.e. the differences in the behavior of the suspension system and the vehicle depending on the selected characteristics of the damper are shown.

Key words: Damper, Asymmetrical damping, Vehicle behavior

\section{INTRODUCTION}

The damping ratio used in passenger vehicles is usually 40 to $60 \%$ higher in the case of bump than in the case of rebound damping, and very rarely can be completely symmetrical. Motorcycle dampers are characterized by even greater asymmetry. Asymmetry may vary depending on the operating conditions of the damper (compression or extension velocity). Sometimes, there is an expressive asymmetry in the dampers placed on the rear axle of passenger vehicles. This phenomenon is much less present with racing vehicles and motorcycles and one of the possible explanations is that greater bump damping, although it leads to reduced comfort, provides a better "sense of the road" to the driver. Scientific research carried out so far has not yielded an acceptable explanation for the existence of asymmetry in dampers. The results of theoretical researches in terms of driving comfort request the dampers to be symmetrical, so this don't provide a valid explanation.

It was found that the asymmetry of the damper is a direct consequence of its construction, i.e. that it is not caused by external influences.

One of the explanations of asymmetry states that the force of gravity which acts downwards on unsprung mass, requires greater damping in extension.

In a case where the wheel has no contact with the road, the gravity force is imposed as a significant influencing factor on the appearance of asymmetrical damping.

It is even stated that the cause of asymmetry is the greater presence of critical holes on the road than the bumps, which can be true, but it's not proven. Even the opposite explanation is offered, which suggests that there are more large bumps so reducing the compression damping is necessary to absorb them, and therefore the reduction in extension damping is not necessary to overcome the large holes on the road. The above claims can be justified only in the case of high compression / extension velocities of the damper. As an influential factor, in the case of previously stated claims, the ability of the tire to "fly" over short holes without the contact with the bottom of the hole is indicated, but a short bump must penetrate the tire profile fully. This argument is important only for small road irregularities.

The question arises - would we have completely symmetrical dampers if the roads would be without holes or bumps?

As a possible cause of asymmetry, it is further stated that passengers in the vehicle do not perceive the same way, in regard to comfort, the various abnormalities of the road.

It is also stated that for damper manufacturers it is more appropriate to have a certain asymmetry and that it is easier to achieve greater damping in the extension than in the compression. Also, there is a greater risk for the occurrence of cavitation of the working fluid in compression stroke [01] and some authors conducted experiments in which is shown that the thermal load of the working fluid has a great influence on damper characteristic [02]. As one of the possible causes of the damper asymmetry it's stated that the damping needs to be higher in the case of extension, since when wheel come across bump, the damper, which is then in the compression stroke, is less involved in the energy absorption impact, this role is primarily taken by the elastic element (spring), all this in order to provide greater passenger comfort. The dissipation of accumulated energy, which takes place after the initial shock or compression of the damper, lasts longer due to the higher damping ratio [03]. The asymmetry of the damper, paired with the nonlinear kinematic response of the suspension system, can significantly change the dynamic response, in particular the return of the vehicle to an equilibrium position [04]. Some authors state that with an increase in asymmetry of the damper, there is also a greater variation of the wheel camber during the vehicle motion, with a non-linear relation with 
the travel of the suspension system [05]. Another conducted research on a real damper used in passenger car shows that real damper characteristic is asymmetric and nonlinear. It states that it is possible to find mean value of damping forces when using symmetric model of damper characteristic, but that it gives much lower values of damping force in extension and much higher values in compression than a real damper [06].

Therefore, the asymmetry of the damper should be considered by taking into account the following parameters:

- road irregularities (bumps, holes, etc.);

- different passenger sensitivity to vehicle movement upwards and downwards;

- asymmetry of the damper as a design characteristic;

- different tire behavior when crossing holes and bumps;

- production costs [01].

This paper analyzes the behavior of a damper as an integral element of the suspension system, as well as the impact of its characteristics on the behavior of the suspension system itself and the whole vehicle. The analysis is focused on telescopic dampers.

\section{RESEARCH ON THE TYPE OF DAMPING AND DAMPING RATIO OF THE DAMPER}

The goal of the study is to examine the influence of the selected characteristics of the damper, i.e. its damping ratio on the behavior of the suspension system and the whole vehicle while moving in different road conditions and in different characteristic maneuvers. This analysis was performed using different values of symmetrical and asymmetrical damping.

Another goal is to make appropriate conclusions on the behavior of the suspension system and the vehicle itself under the above mentioned conditions, i.e. the effect of damper characteristic change on handling, stability and comfort.

The effect of damping ratio change on the behavior of the suspension system and the vehicle was analyzed using the CarSim 8 software. For the needs of the simulation, a $\mathrm{C}$ segment vehicle is choosen with a sprung mass of $1274 \mathrm{~kg}$ and unsprung mass of $142 \mathrm{~kg}$. The simulation of each of the selected tests is performed for four cases, each with a different characteristic of the damper.

The change in damper characteristic was made by changing the shape of the curve that shows damping force versus the velocity of the damper piston. The shape of the curve should be as smooth as possible to eliminate problems in exploitation and unnatural sensation to the driver.

The shape of the curve used in the first case is shown in Figure 1 and represents an initial asymmetrical damping.

Based on the diagram shown, it can be concluded that the bigger damping is present in the extraction (bottom left quadrant) than in the damper compression (upper

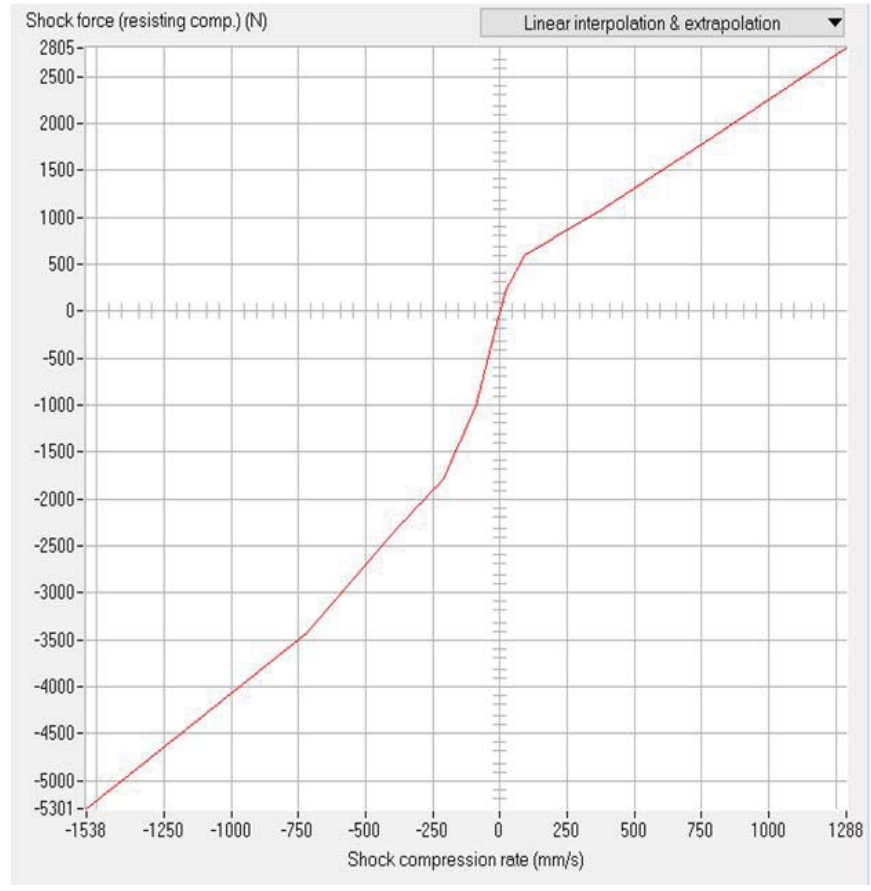

Figure 1: Initial asymmetrical damping

right quadrant). This is in favor of the previously stated fact that the asymmetry is often present, and the characteristic used in this case is a basic setting for the subject vehicle, given by the software.

For the second case, the symmetrical characteristic of the damper is chosen, so that the extreme damping values of compression and extraction force correspond to the extreme values of damping force in compression stroke, as showed in Figure 1.

For the third case, the symmetrical characteristic of the damper was also chosen, but with the extreme values of the compression and extraction corresponding to the extreme values of damping force in extraction stroke, as showed in Figure 1.

For the fourth case, the reverse asymmetrical characteristic of the damper was chosen, in which the damping is bigger in compression. This case is only theoretical, because it is not usually present in exploitation, but it is necessary to analyze it.

In all four cases, a different curve slope is present for low and high piston velocity. At lower piston rates, damping force increases more rapidly compared to higher rates. The first is to improve vehicle stability, and the second to better absorb the road irregularities.

Five different tests were performed with each of the damper settings described above.

\section{Test 1}

The first conducted test is a comfort test, and it is derived in two parts. In the first part of the test, the vehicle moves on a wavy road section with initially lower frequency, which increases later. The vehicle moves at a constant 
speed of $40 \mathrm{~km} / \mathrm{h}$. The road profile is shown in Figure 2. In the second part of the test, the vehicle moves along a cross-slope section, with a constant speed of $20 \mathrm{~km} / \mathrm{h}$.

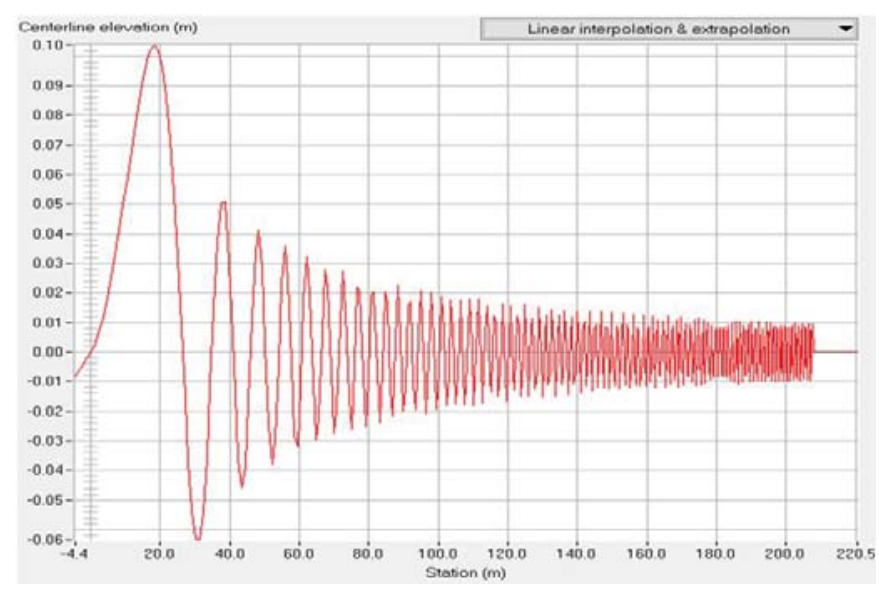

Figure 2: Road profile of the selected road section

Figure 3 shows the road profile for this part.

\section{Test 2}

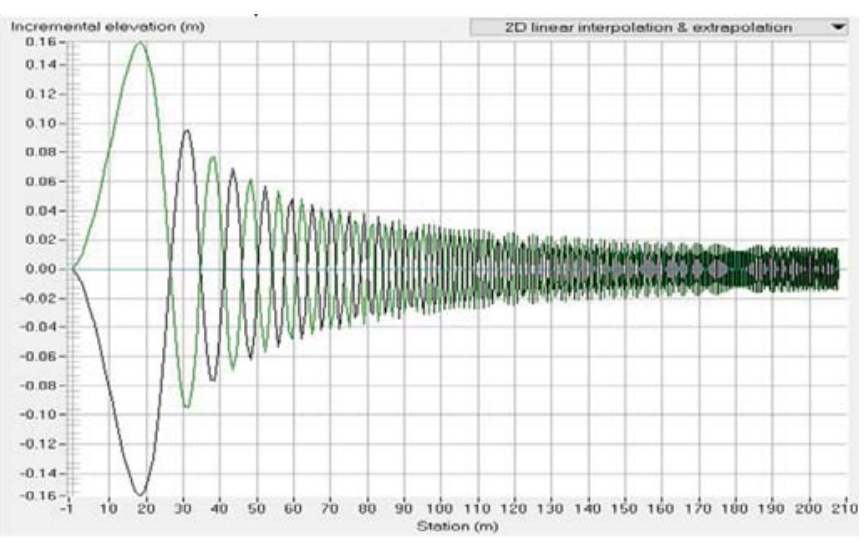

Figure 3: Profile of the cross-slope road section

For the second test, the fishhook test is chosen and the nature of vehicle motion during the test is shown in Figure 4.

\section{Test 3}

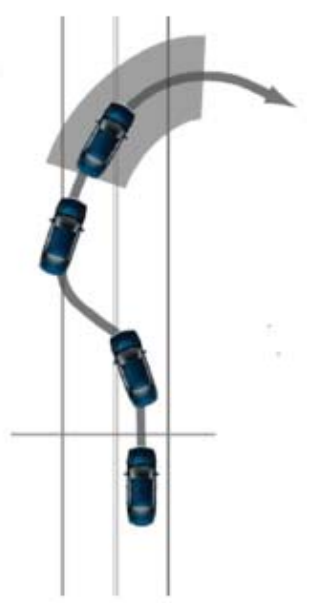

Figure 4: Fishhook test
The DLC (Double Lane Change) test was selected for the third test. The vehicle moves at a constant speed of $120 \mathrm{~km} / \mathrm{h}$. The nature of vehicle movement during this test, as well as the required dimensions of the test track are shown in Figure 5.

\section{Test 4}

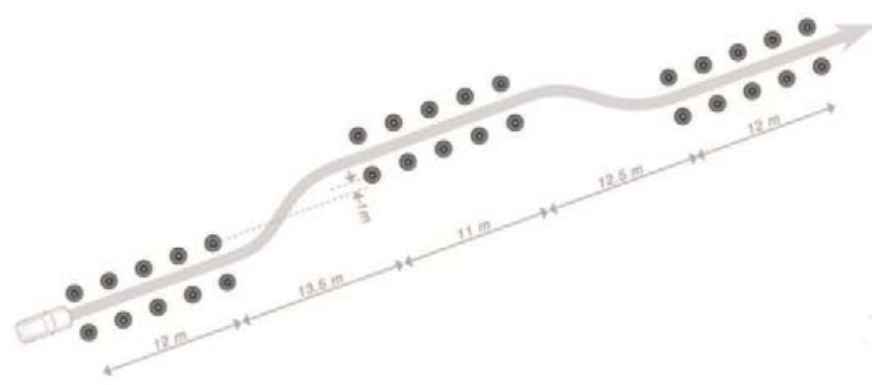

Figure 5: Double Lane Change test

In this test, the vehicle is crossing over a short transverse road bump is chosen as the fourth test. The vehicle moves at a constant speed (first at 40 and later at 80 $\mathrm{km} / \mathrm{h}$ ) and crosses the transverse bump that is $35 \mathrm{~mm}$ high and $400 \mathrm{~mm}$ long.

\section{Test 5}

For the fifth test, the vehicle moves at a constant speed of $50 \mathrm{~km} / \mathrm{h}$ along an uneven $1200 \mathrm{~m}$ long road with small irregularities. The profile of the selected road section is given in Figure 6.

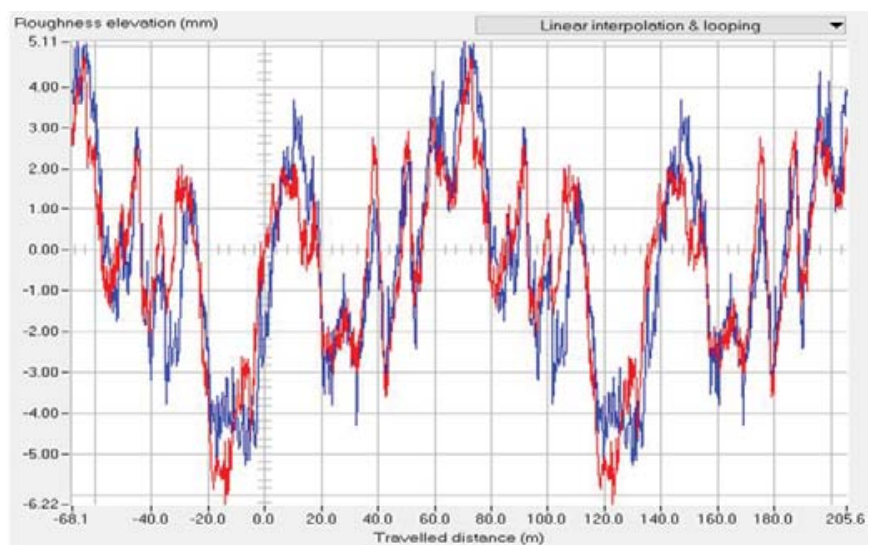

Figure 6: Road profile

\section{THE ANALYSIS OF THE RESULTS}

\section{Test 1 - first part}

Figure 7 shows the vertical acceleration of the vehicle's center of gravity for each of the four selected damper characteristics. 


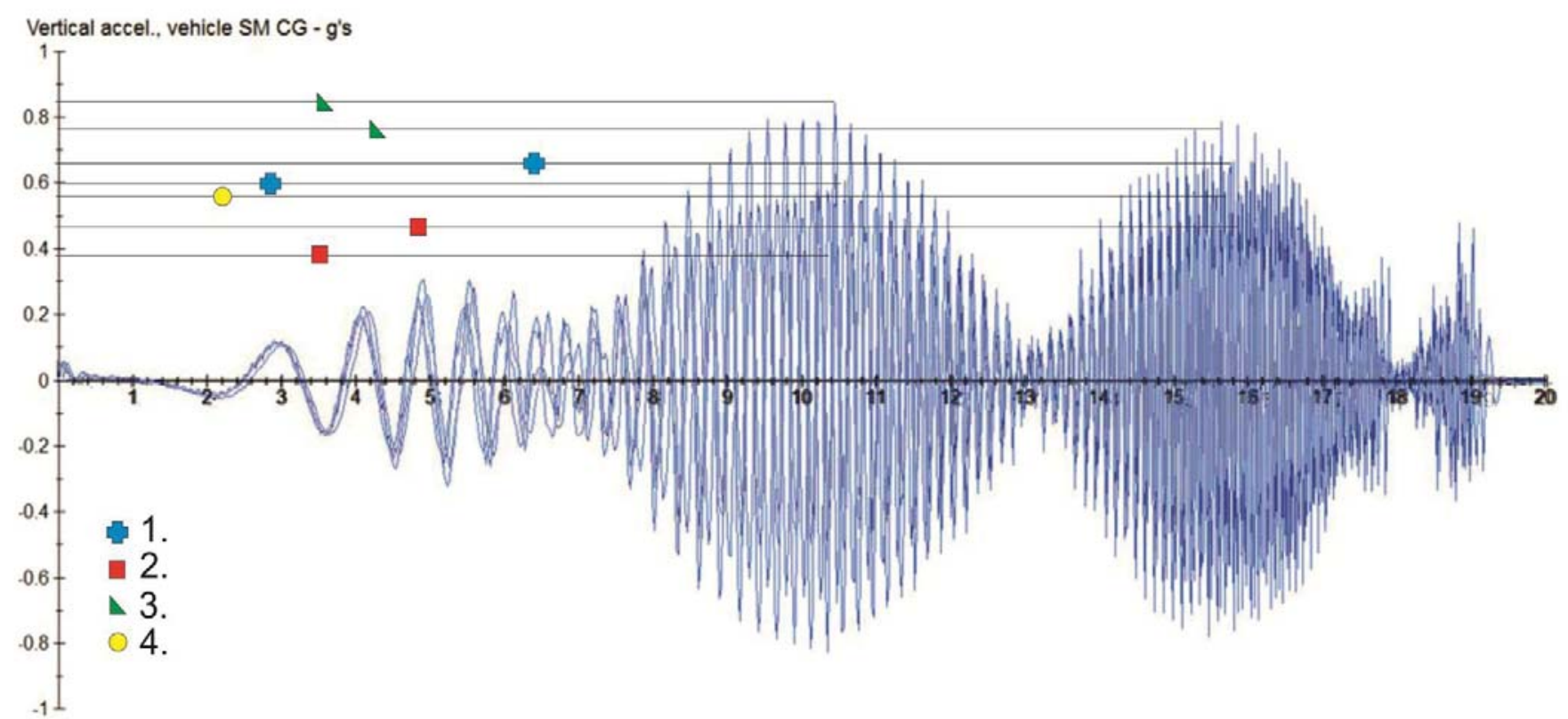

Time - sec

Figure 7: Vertical acceleration of the vehicle's CG

In the first part, where the frequency of oscillations is the lowest, most favorable vertical acceleration of the center of gravity of the sprung mass is present in the third case, when there is a higher value of the symmetrical damping.

The initial asymmetrical damping and lower symmetrical damping provide approximately the same change in vertical acceleration of the vehicle's center of gravity, with somewhat less extreme values achieved (initial asymmetrical damping).

Reverse asymmetrical damping ensures a change in vertical acceleration of a vehicle whose extreme values correspond to asymmetrical damping, but in the opposite direction. With reverse asymmetrical damping, there are certain unevennesses in the change of vertical acceleration, which are not present in other cases.

Observing the second part, where the frequency of the oscillations is increased, the most favorable vertical acceleration of the vehicle is present in the second case, hence the lower value of the symmetrical damping here gives the best results. The initial asymmetrical damping leads to somewhat higher vertical acceleration, while higher symmetrical damping value causes a significantly higher vertical acceleration.

Reverse asymmetrical damping provides maximum acceleration values for the vehicle's center of gravity between the values obtained by lower symmetrical and initial asymmetrical damping.

By analyzing the third part, where the frequency of oscillations is the highest, most favorable vertical acceleration of the center of gravity of the sprung mass is also present in the second case, therefore, the lower value of the symmetrical damping gives the most favorable results. The initial asymmetrical damping again results in slightly higher vertical acceleration, while vertical acceleration achieved by the reversed asymmetrical damping is smaller. A higher symmetrical damping value causes a significantly increased vertical acceleration of the vehicle's center of gravity. At a higher value of symmetrical damping, a smaller oscillation amplitude is present in the third part relative to the second part, while in the first two cases it is the opposite.

In the case of reversed asymmetrical damping, the extreme values of vertical acceleration of the vehicle are almost identical in the second and third part of the chosen road section.

A conclusion can be drawn that the lower symmetrical damping value gives the best results for such conditions of vehicle movement, which does not necessarily mean that the vehicle with this type of damper will provide the most favorable behavior in the various maneuvers and driving conditions.

It can be noticed that in the case of asymmetrical damping, the damper predominantly moves in the compression stroke, while movement in the extension part is present only on several occasions when the vehicle moves on a road section with a lower frequency of "waves". A totally opposite situation of the above is present in the case of a damper with a reversed asymmetrical damping characteristic.

In other cases this movement is almost symmetrical throughout the test. The highest value of the piston movement is achieved by the compression of the damper with asymmetrical damping, and the lowest in the extension. It can be concluded that a higher damping value results in less movement of the damper piston. 
On the basis of conducted research, it can be concluded that with symmetrical damping, the displacement of suspension system is symmetrical upwards and downwards, while initial asymmetrical damping increases the upward movement. When using the reverse asymmetrical characteristic of the damper, the downward movement of the suspension system is increased. The total travel of the suspension system is smallest when using both asymmetrical characteristics of the damper, which can lead to a reduction in tire and suspension system elements wear.

\section{Test 1 - second part}

Figure 8 shows vertical acceleration of the center of gravity of the vehicle. By looking at diagrams shown, it is noted that most unfavorable results for the comfort of the passengers (the highest values of the vertical acceleration of the vehicle) are achieved with a damper with an asymmetrical damping characteristic. The initial asymmetrical damping yields less favorable results than the reversed asymmetrical characteristic. The most favorable results are achieved by selecting a damper with a lower symmetrical damping value, while a higher symmetrical damping value provides slightly higher values of vertical acceleration of the vehicle's center of gravity.

On the basis of the obtained diagrams, it can be concluded that the damping characteristics do not have a significant role in reducing the roll of the vehicle, since the results achieved in all four cases are almost identical.

Figure 9 shows the pitch of the vehicle during the test. It can be noticed that the change in vehicle pitch with two symmetrical damping characteristics is very similar, as opposed to asymmetrical damping. Obtained extreme value of pitch is almost the same in first three cases, while maximum value achieved with the last case is a little bit higher. In the case of initial asymmetrical damping, the pitch of the vehicle changes the direction, whereas in the case of symmetrical damping this is not the case.

Reverse asymmetrical damping causes an additional increase in pitch, especially in the final stage of the test.

\section{Test 2}

On the basis of the obtained results, it can be seen that maximum values of the achieved yaw rate of the vehicle in all three cases range from 25 to $30 \%$.

At the beginning of the test there was a slightly higher yaw rate of the vehicle, when dampers with a lower symmetrical damping value are selected, while maximum yaw rate, after changing the direction of movement, is provided by dampers with a higher value of symmetrical and reverse asymmetrical damping. The difference in the nature of the change of the yaw rate is present, which shows that the choice of the damper characteristics and the maximum damping ratio is certainly important for a sudden change of the direction (performed in the first part of the test), while in the second part of the fishhook test, when the vehicle is moving in a constant radius, there are almost no differences in the behavior of the vehicle.

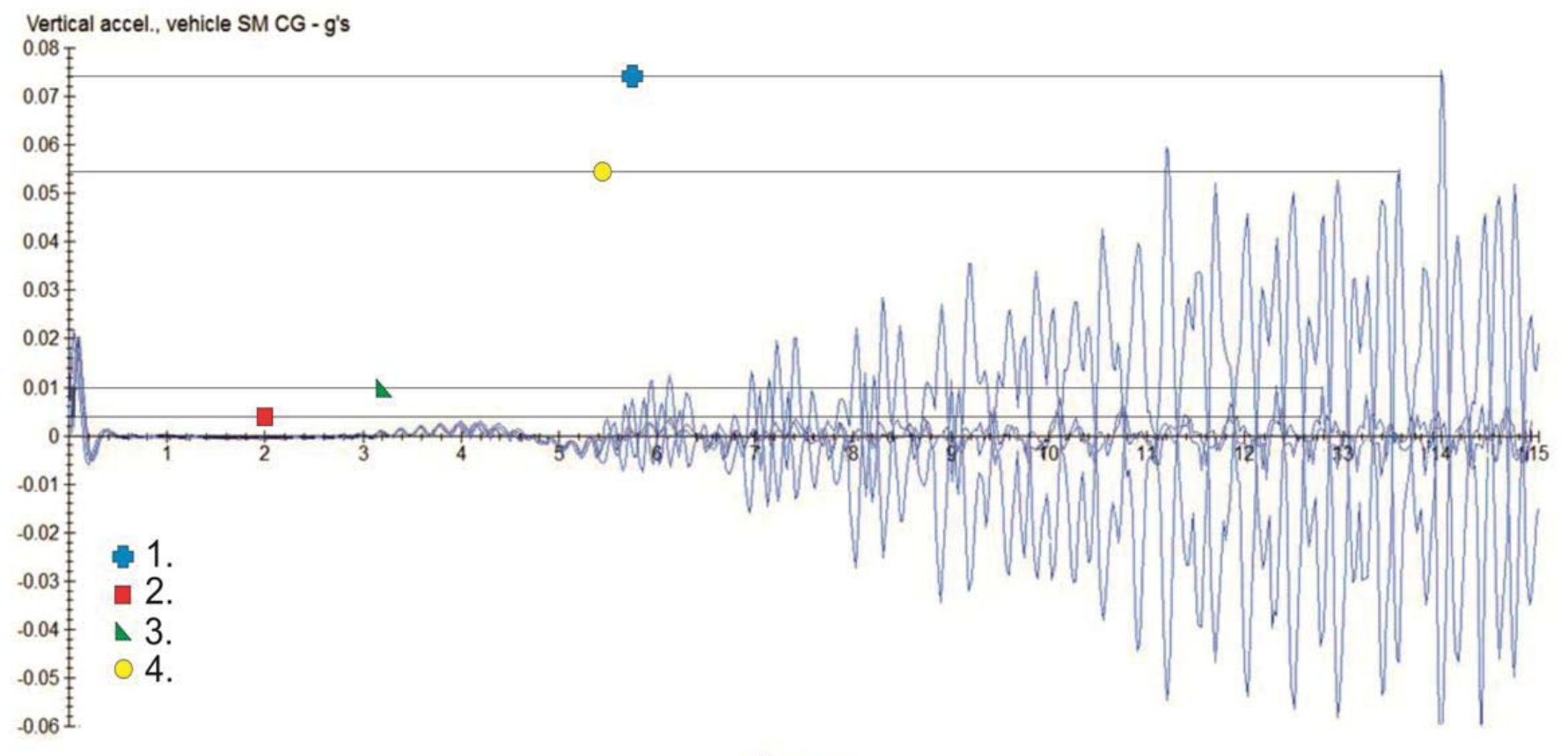

Figure 8: Vertical acceleration of the vehicle's CG 
It can be seen that the maximum value of lateral force in contact between the tire and the road in the case of symmetrical and asymmetrical characteristics of the damper is same in the first part of the performed fishhook test, but there is a difference in the way this force changes. In the second part of the fishhook test, when a second direction change takes place and vehicle continues to move without sudden changes in direction, the lowest value of lateral force is achieved when using a damper with a higher symmetrical damping value, while the highest value is achieved by the dampers with initial asymmetrical damping.

Dampers with a lower symmetrical damping value cause lateral forces whose intensity is between the afore mentioned damping characteristics.

The lateral force change over time has the most pronounced jumps or unevennesses in the fourth case, while the lower value of the symmetrical damping also leads to the appearance of certain unevennesses which are less pronounced than in the afore mentioned case.

\section{Test 3}

In all four cases, when an identical lateral deviation of the vehicle from the desired path was obtained and because of that it is not shown. This tells us that the damping ratio of the damper is not of great importance when the vehicle is found in such driving conditions, but primarily comes the stiffness of the springs as well as the geometric and mass parameters of the vehicle.

\section{Test 4}

Figure 10 shows the vertical acceleration of the vehicle when the vehicle passes the transversal bump at a speed of $40 \mathrm{~km} / \mathrm{h}$.

It can be seen from the Figure 10 that extreme values achieved here in all four cases are almost identical, while the change in the vertical acceleration of the vehicle itself has certain variations depending on the selected damper characteristic. We can conclude that in all four cases the comfort is approximately at the same level, regardless of the chosen damper characteristic.

The extreme value of the longitudinal force is approximately the same in the case of initial asymmetrical damping and lower symmetrical damping whereas it is significantly lower at higher values of symmetrical damping. The lowest value of the longitudinal force is present in the reverse asymmetrical damping which can be considered the most favorable case. The change in the vertical force is approximately equal in all four cases, with identical extreme values achieved.

Figure 11 shows the vertical acceleration of the vehicle when the vehicle passes the transversal bump at a speed of $80 \mathrm{~km} / \mathrm{h}$.

Based on Figure 11, it is again possible to conclude that the extreme values achieved here in all four cases are almost identical, while the change in vertical acceleration of the vehicle itself also has certain variations depending on the selected damping characteristic. Again, we can conclude that in all four cases, the impact on the passengers, i.e. the achieved comfort, is approximately at the same level, regardless of the selected damping characteristic.

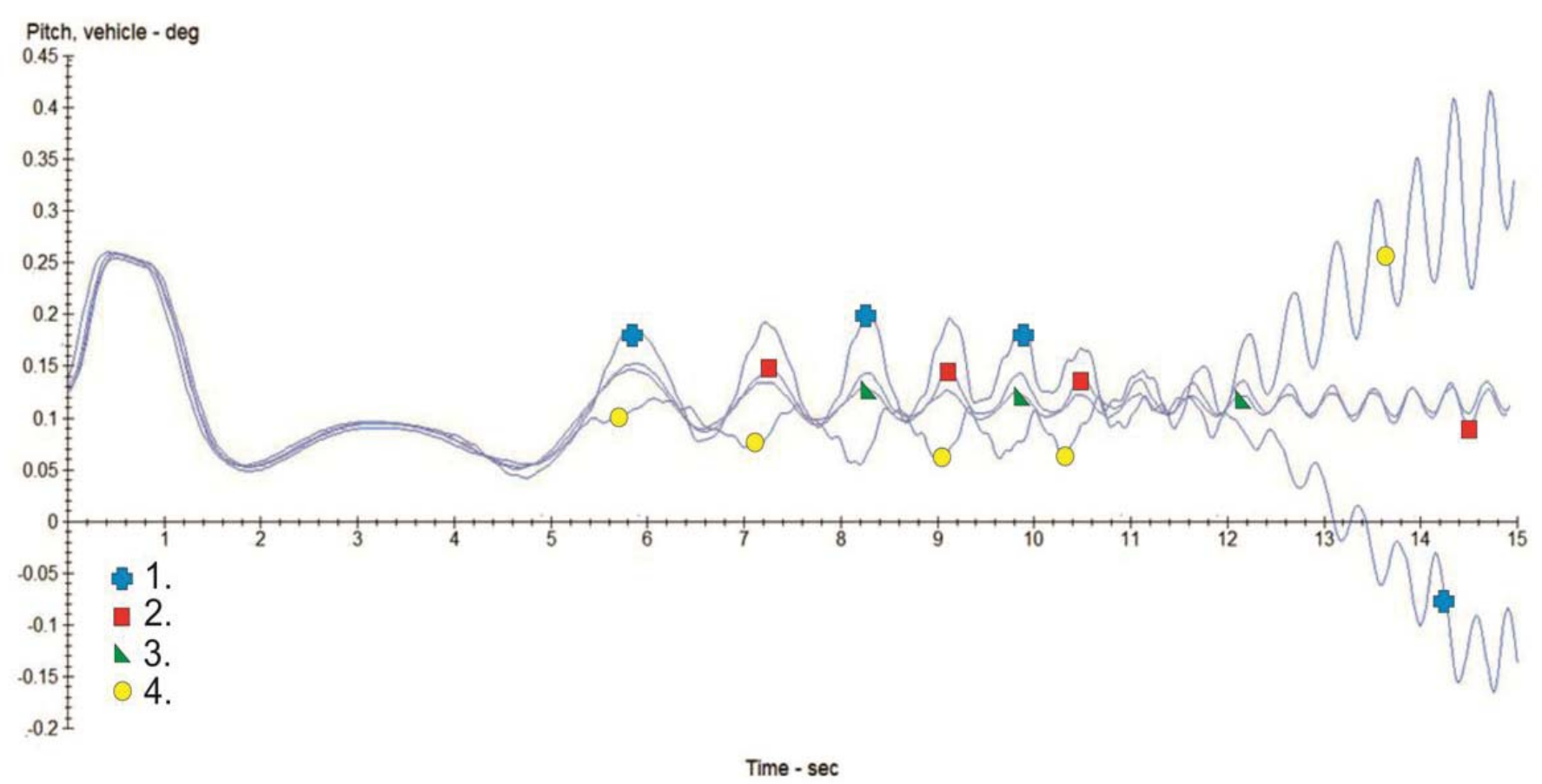

Figure 9: Vehicle pitch 
By analyzing the achieved results of the research for different characteristics of the damper, it is possible to derive conclusions, so for the purpose of comparative analysis, the results achieved for each of the tests are evaluated and shown in the table.

The scores for each of the criteria considered during the performed tests are given in the range of 1 to 4 points (the best result achieved being 4 , and the worst one being 1 point) for each of the selected dampers and are shown in Table 1. For tests performed at two vehicle speeds, the results for each of the considered criteria are given in the following format - the number of points achieved at the first speed value / number of points achieved at the second speed value, while the total score for each of the criteria is shown as the average number of points and is given in parentheses.

Corresponding weight factors were selected for each of the tests and the criteria considered. On the basis of the stated weight factors, the significance of each test, as well as the significance of each of the criteria within the selected tests is defined.

By assigning the appropriate points to each of the considered damper characteristics for each of the performed tests, almost equal results are obtained.

By analyzing the first part of test 1 , it is concluded that when moving along the wavy road, the best results are obtained with the reverse asymmetrical damping which, as previously mentioned, is not present in the exploitation. This damping characteristic provides even better results in terms of vertical acceleration and pitch.

The lower symmetrical damping value gives slightly worse overall results for the subject test.

Although it provides the best results in terms of vertical acceleration, it is proving to be the worst possible solu- tion for the vehicle pitch.

The initial asymmetrical damping ensures uniform behavior in terms of vertical acceleration and pitch of the vehicle, but in the overall score for the considered test it is worse than the lower symmetrical damping.

A higher symmetrical damping value gives the most unfavorable vertical acceleration while obtaining low pitch values. The achieved overall result for the test is at the level of initial asymmetrical damping.

In the second part of test 1 , with the movement of the vehicle on the road with a cross-slope, the behavior of a vehicle with symmetrical damping characteristics is noticeably more favorable than a vehicle with asymmetrical dampers.

Both symmetrical damping values provide very good results in terms of reducing the vehicle roll. A lower symmetrical damping value provides a more favorable vertical acceleration of the vehicle's center of gravity, while higher symmetrical damping value provides a more favorable mitigation of the pitch.

By comparing asymmetrical characteristics, identical conclusions arise as well as in the case of symmetrical characteristics of the dampers, but it should be noted that the overall results achieved are less favorable.

In fishhook test, the vehicle achieved identical results for each of the selected characteristics, but there are various changes in lateral and vertical forces in the contact of the tire and the road. Also, the obtained maximum values of these forces are not the same. This leads to different tire deformations which certainly have a significant impact on its behavior. Bearing in mind that the tire, primarily it's elastic characteristics, participates in the operation of the suspension system, the study of these effects requires additional attention in some future research.

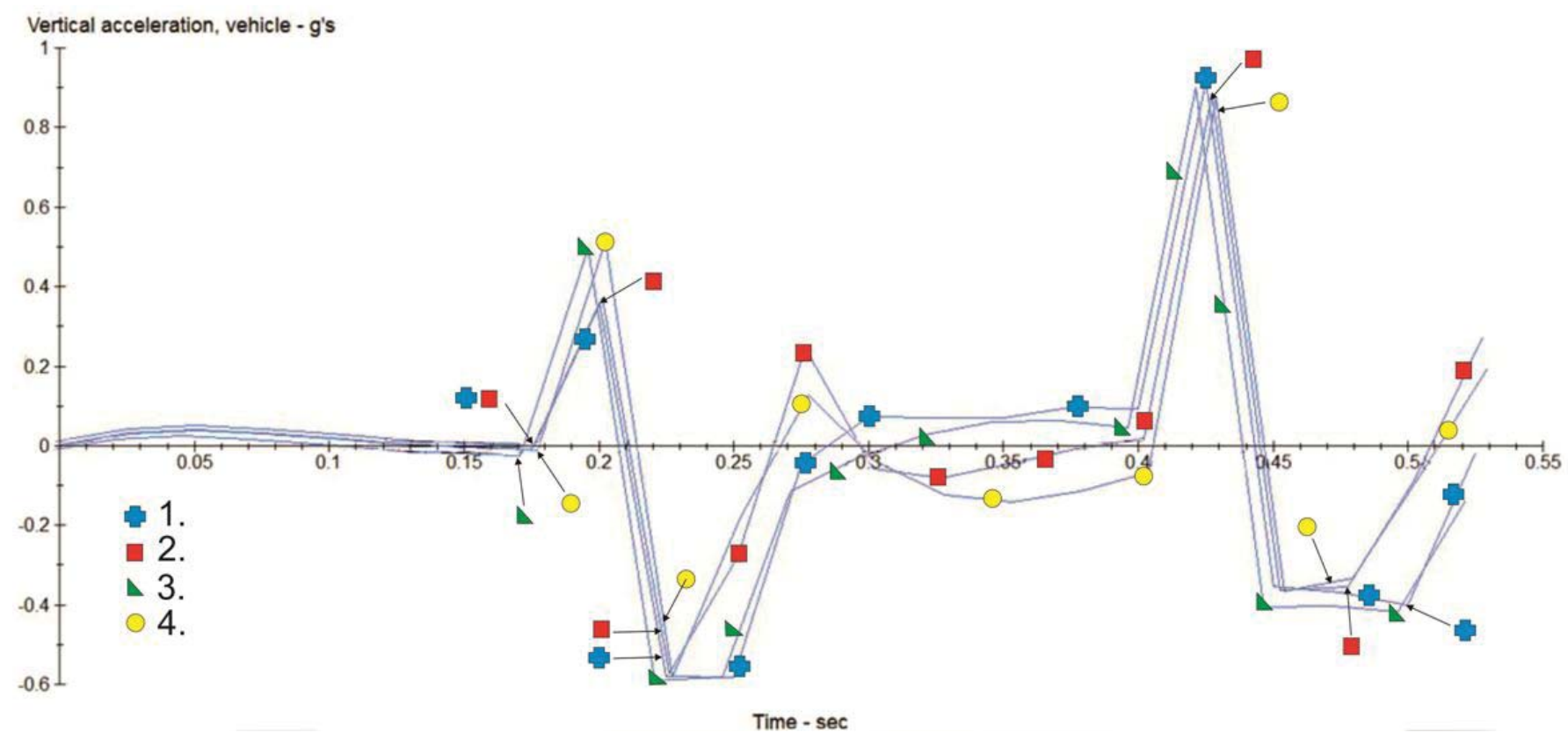

Figure 10: Vertical acceleration of the vehicle 
Test 3 (DLC test) shows that vehicle handling is at the same level with all selected damper characteristics. Due to the low impact of the damper on the steering behavior of the vehicle and the absence of differences in the parameters that depend on the behavior of the suspension system (vertical acceleration of the vehicle, pitch, etc.), this test is assigned a weight factor of 0.5 .

When vehicle crosses a transversal bump (test 4 ), the higher symmetrical and reversed asymmetrical damping give completely opposite results with two different speeds of vehicle. Higher symmetrical damping value is shown to be a more unfavorable solution at lower

speeds, while at higher speeds it is shown to be the most favorable. The completely opposite conclusion is made for reversed asymmetrical damping. The initial asymmetrical damping and lower symmetrical damping value show solid results at each of the selected speeds.

The overall results for this test show that each of the characteristics has been shown to be almost the same, but when selecting the optimum characteristic it should be kept in mind that the vehicle's favorable behavior is ensured in the broader range of speed and road characteristics (bumps, holes, etc).

Differences in the achieved amplitude of the vertical acceleration, as well as the achieved frequency when the vehicle is moving through a road with the small unevennesses, are barely noticeable.

\section{CONCLUSION}

Summing up all the results leads to the conclusion that there are no dramatic differences in the behavior of the vehicle depending on the choice of the damper characteristic. It can be said that the obtained results are fairly uniform and that the application of any type of damping characteristic for the vehicle under consideration will not lead to significant disturbance of the comfort or stability of the vehicle.

Reverse asymmetrical damping characteristic surpasses all the expectations, since such solution is not present in practice. In some segments it turned out even better than other configurations.

In the future, there is also a need to analyze cases with a higher mass of vehicle and to further investigate the allegations [03] that asymmetry can only be present on one of the axles, and that in this way the removal of vehicle vibrations is ensured and that it is almost at the same level as for vehicles using asymmetrical dampers on both axles.

In order to fully understand the phenomenon of asymmetry, it is necessary to examine in detail the construction of the damper itself, as well as the influence of the choice of the damping characteristics on the costs of development and production of dampers.

In practice, there are semi-active suspension systems that have the ability to change the damper characteristic in order to achieve a high level of comfort while maintaining the stability of the vehicle under different driving conditions. The damper characteristic can be changed by adjusting the viscosity of magnetorheological or electrorheological fluid inside the damper, which provides a higher or lower value of damping ratio.

\section{ACKNOWLEDGEMENT}

This paper presents some of the results obtained through the project supported by Serbian Ministry of Education, Science and Technological Development (TR 35045 "Scientific-Technological Support to Enhancing the Safety of Special Road and Rail Vehicles").

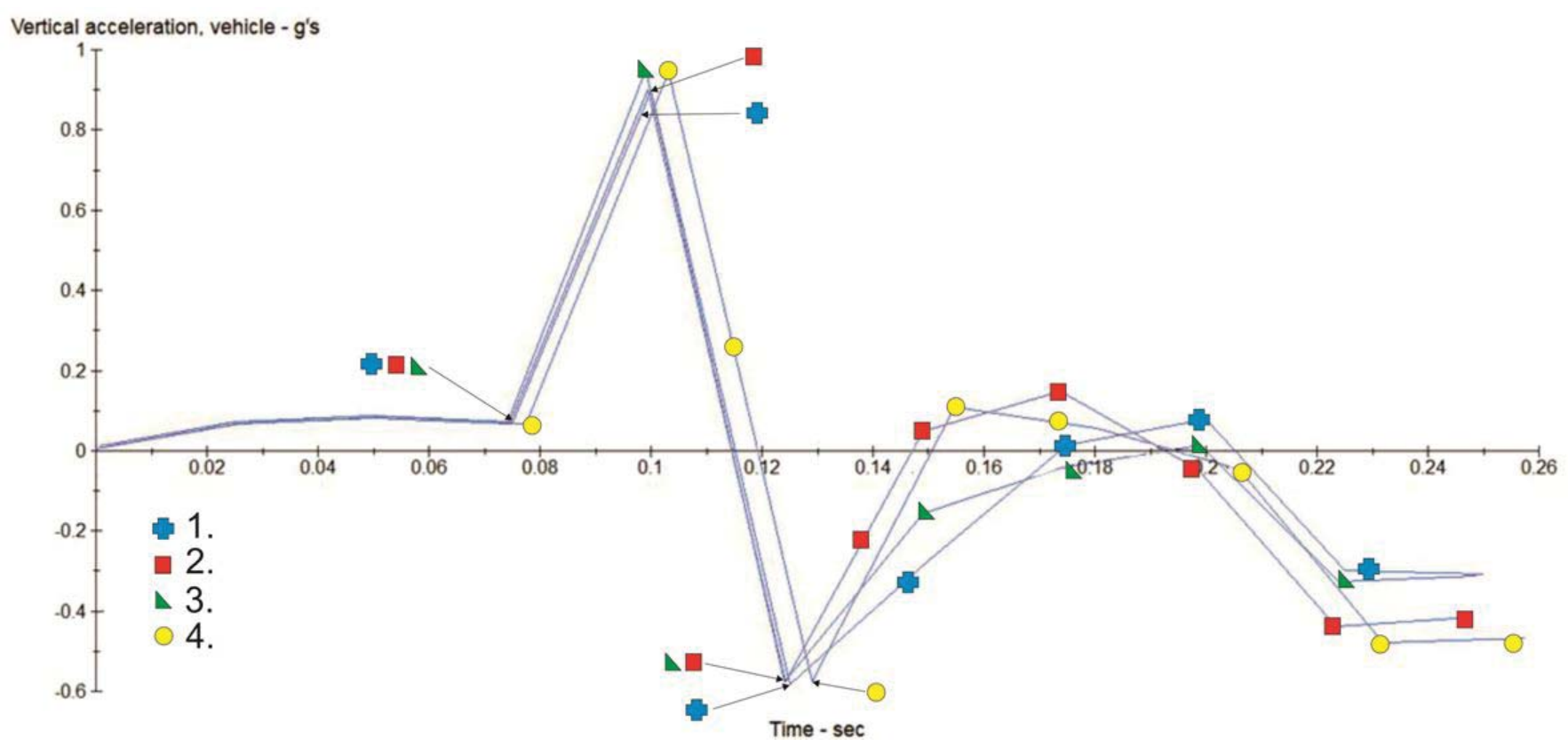

Figure 11: Vertical acceleration of the vehicle 
Table 1: Evaluation of the results of the survey

\begin{tabular}{|c|c|c|c|c|c|c|}
\hline $\begin{array}{l}\text { Weight factor } \\
\text { of the test }\end{array}$ & Criterion & $\begin{array}{l}\text { Weight factor } \\
\text { of the criterion }\end{array}$ & $\begin{array}{c}\text { Initial } \\
\text { asymmetrical } \\
\text { damping }\end{array}$ & $\begin{array}{c}\text { Lower } \\
\text { symmetrical } \\
\text { damping }\end{array}$ & $\begin{array}{c}\text { Higher } \\
\text { symmetrical } \\
\text { damping }\end{array}$ & $\begin{array}{c}\text { Reverse } \\
\text { asymmetrical } \\
\text { damping }\end{array}$ \\
\hline \multicolumn{7}{|c|}{ Test 1 - first part } \\
\hline \multirow{3}{*}{1} & $\begin{array}{c}\text { Vertical } \\
\text { acceleration }\end{array}$ & 0.5 & 2 & 4 & 1 & 3 \\
\hline & Pitch & 0.5 & 2 & 1 & 3 & 4 \\
\hline & Total & 1 & 2 & 2.5 & 2 & 3.5 \\
\hline \multicolumn{7}{|c|}{ Test 1 - second part } \\
\hline \multirow{4}{*}{1} & $\begin{array}{c}\text { Vertical } \\
\text { acceleration }\end{array}$ & 0.33 & 1 & 4 & 3 & 2 \\
\hline & Roll & 0.33 & 4 & 4 & 4 & 4 \\
\hline & Pitch & 0.33 & 2 & 3 & 4 & 1 \\
\hline & Total & 1 & 2.33 & 3.67 & 3.67 & 2.33 \\
\hline \multicolumn{7}{|c|}{ Test 2} \\
\hline \multirow{2}{*}{1} & Stability & 1 & 4 & 4 & 4 & 4 \\
\hline & Total & 1 & 4 & 4 & 4 & 4 \\
\hline \multicolumn{7}{|c|}{ Test 3} \\
\hline \multirow[t]{2}{*}{0.5} & $\begin{array}{l}\text { Lateral offset } \\
\text { from } \\
\text { designed path }\end{array}$ & 1 & 4 & 4 & 4 & 4 \\
\hline & Total & 1 & 4 & 4 & 4 & 4 \\
\hline \multicolumn{7}{|c|}{ Test 4} \\
\hline \multirow{2}{*}{1} & $\begin{array}{c}\text { Vertical } \\
\text { acceleration }\end{array}$ & 1 & $2 / 3(2.5)$ & $3 / 2$ (2.5) & $1 / 4(2.5)$ & $4 / 1$ (2.5) \\
\hline & Total & 1 & 2.5 & 2.5 & 2.5 & 2.5 \\
\hline \multicolumn{7}{|c|}{ Test 5} \\
\hline \multirow{2}{*}{1} & $\begin{array}{c}\text { Vertical } \\
\text { acceleration }\end{array}$ & 1 & 4 & 4 & 4 & 4 \\
\hline & Total & 1 & 4 & 4 & 4 & 4 \\
\hline \multicolumn{3}{|c|}{ TOTAL POINTS } & 18.83 & 20.67 & 20.17 & 20.33 \\
\hline
\end{tabular}

\section{REFERENCES}

1. Dixon, J.C. (2007). The shock absorber handbook (2nd ed). John Wiley and Sons, Chichester.

2. Demić, M., Diligenski, Đ., Milovanović, M. (2013). A contribution to research of the influence of degradation of vehicle vibration parameters on thermal load of shock absorbers. Journal of Applied Engineering Science, vol. 11, no. 1, 23-30.

3. Silveira, M., Pontes Jr., B.R., Balthazar, J.M. (2014). Use of nonlinear asymmetrical shock absorber to improve comfort on passenger vehicles. Journal of Sound and Vibration, vol. 333, no. 7, 2114-2129, DOI:10.1016/j.jsv.2013.12.001.

4. Balike, K.P. (2010). Kineto-dynamic analyses of vehicle suspension for optimal synthesis (PhD thesis).
Concordia University, Montreal.

5. Balike, K.P., Rakheja, S., Stiharu, I. (2010). Optimization of asymmetric damper parameters of an automotive suspension for minimal camber angle variations. 12th International Conference on Advanced Vehicle and Tire Technologies, p. 57-64.

6. Ślaski, G. (2011). Damping parameters of suspension of passenger vehicle equipped with semi-active dampers with by-pass valve. Transport Problems, vol. 6 , no. 2, 35-42.

Paper submitted: 04.05.2018.

Paper accepted: 25.07.2018.

This is an open access article distributed under the CC $B Y-N C-N D 4.0$ terms and conditions. 